\title{
The Akt pathway in human breast cancer: a tissue-array-based analysis
}

\author{
Shikha Bose ${ }^{1,3}$, Sindhu Chandran ${ }^{1}$, James M Mirocha ${ }^{2}$ and Namrata Bose ${ }^{1}$ \\ ${ }^{1}$ Department of Pathology, Cedars Sinai Medical Center, Los Angeles, CA, USA; ${ }^{2}$ Biostatistics Core and \\ Research Institute, Cedars Sinai Medical Center, Los Angeles, CA, USA and ${ }^{3}$ David Geffen School of \\ Medicine, UCLA, Los Angeles, CA, USA
}

\begin{abstract}
The Akt pathway, an important regulator of cell proliferation and survival, is deregulated in many cancers. The pathway has achieved considerable importance due to the development of kinase inhibitors that are able to successfully reduce tumor growth. This study was conducted to determine the status of the Akt pathway in human breast cancers and to study the relationship between the different component proteins. Expression levels of PTEN, phosphorylated forms of the constituent proteins (Akt, FKHR, mTOR, and S6) and cyclin D1 were evaluated by immunohistochemistry, on consecutive sections from a tissue microarray containing 145 invasive breast cancers and 140 pure ductal carcinomas in-situ. Aberrant expression was correlated statistically with tumor characteristics and disease outcome. The Akt pathway was found to be activated early in breast cancer, in the in-situ stage. In all, 33, 15, 32, and $60 \%$ of ductal carcinoma in-situ showed overexpression of Akt, FKHR, mTOR, and cyclin D1. PTEN loss did not correlate statistically with expression of AKT or any of the other proteins with the exception of S6, indicating that Akt activation was not a result of PTEN loss. Expression levels of PTEN and S6 were significantly different in in-situ and invasive cancers, indicating association with disease progression. Loss of PTEN was noted in $11 \%$ of in-situ as compared to $26 \%$ of invasive cancers, while $\mathbf{S 6}$ overexpression was seen in $47 \%$ in-situ and in $72 \%$ invasive cancers. High-grade carcinomas were associated with PTEN loss, while low-grade carcinomas with good prognostic features showed cyclin D1 overexpression and were associated with longer disease free survival. Additionally, cancers with mTOR overexpression showed a three times greater risk for disease recurrence. Overall, a large proportion of in-situ and invasive breast cancers overexpressed cyclinD1 and S6. Our results may have significant implications in the development and application of targeted therapy.
\end{abstract}

Modern Pathology (2006) 19, 238-245. doi:10.1038/modpathol.3800525; published online 2 December 2005

Keywords: PTEN; AKT pathway; mTOR; cyclin D1; human breast cancer; tissue array

The Akt pathway regulates many diverse biological functions, including cellular proliferation, survival, and motility. ${ }^{1}$ The components of the Akt pathway are deregulated in a wide spectrum of human cancers with gain-or loss-of-function mutants leading to neoplastic transformation. This pathway has generated considerable interest in recent years since its various components provide attractive targets for therapy and several inhibitors are being developed and tested in early clinical trials. ${ }^{2,3}$

The pathway is activated by receptor tyrosine kinase growth factors that lead to the generation of membrane-bound phosphoinositides which then act

Correspondence: Dr S Bose, MD, Department of Pathology, South Tower \#8732, Cedars Sinai Medical Center, 8400 Beverly Blvd., Los Angeles, CA 90048, USA.

E-mail: BoseS@cshs.org

Received 8 July 2005; revised and accepted 18 October 2005; published online 2 December 2005 as second messengers to recruit and activate Akt (Figure 1). Phosphorylation of Akt results in the activation of a cascade of different protein targets involved in apoptosis, cell growth, and proliferation, all of which promote tumorigenesis. ${ }^{4-8}$ PTEN is a lipid phosphatase that opposes the ability of phosphatidylinositol 3-kinase (PI3K) to phosphorylate Akt, ${ }^{9,10}$ thereby functioning as a tumor suppressor. Although constitutive activation of Akt and/or PI3K is described in a growing list of solid tumors as a mechanism for transformation, ${ }^{11-15}$ the most frequently mutated component of this pathway, by far, is PTEN, found to have an average mutation rate of $16 \%$ across many tumor types, to as high as $83 \%$ in endometrial cancers. ${ }^{16}$ We have previously demonstrated that the PTEN protein is lost/reduced in $38 \%$ of human invasive breast cancers. ${ }^{17}$ Our understanding of the signaling events that occur downstream of PTEN derives primarily from experimental models. Initial clinical studies suggest that not only 


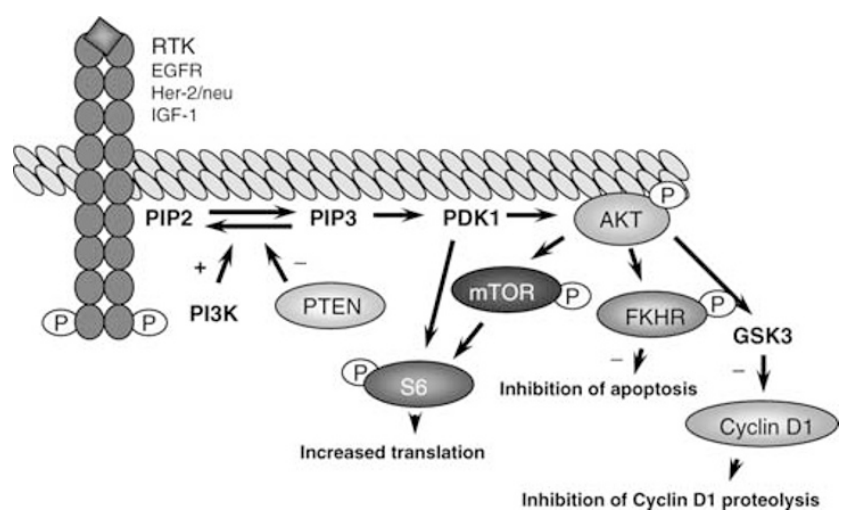

Figure 1 Diagrammatic representation of the Akt pathway. RTKreceptor tyrosine kinase, EGFR-epidermal growth factor receptor, IGF-1 - insulin-dependent growth factor, PIP2/3-phosphatidylinositol 4,5-/3,4,5-phosphate, PI3K-phosphatidylinositol 3-kinase, PDK1-3-phosphoinositide-dependent protein kinase1, GSK3-glycogen synthase kinase-3 $\beta$.

would patients benefit from use of PI3K pathway inhibitors in breast cancer, but also that PTEN loss is critical to tumorigenesis and modulates response to therapy. ${ }^{18}$ The study was undertaken to analyze this pathway in human breast cancers and validate experimental data. This has important implications for designing effective clinical trials and selecting the right patients for molecular therapy. A tissue array of human breast cancers was chosen for this purpose since it provides an effective means of testing large samples of cancers and also allows accurate analysis of relationships between different proteins.

\section{Materials and methods}

\section{Human Breast Cancer Tissue Microarray}

Tissue microarrays were constructed using a manual arrayer (Beecher Instruments, MD, USA) from 285 cases of breast carcinoma diagnosed at the Department of Pathology and Laboratory Medicine at Cedars-Sinai Medical Center, Los Angeles, from 1991 to 1998, after approval from the Institutional Review Board. Cases included 145 invasive carcinomas and 140 ductal carcinomas in situ (DCIS) without associated invasive carcinoma. Clinical parameters and follow-up information were obtained from hospital records. Three 1-mm cores of invasive cancer or DCIS and adjacent normal glandular tissue were arrayed from each case. DCIS cases were mostly of intermediate (39\%) or high nuclear grade (47\%); $27 \%$ were of the comedo subtype. Invasive carcinomas were predominantly ductal (96\%), varying in size from $<0.1$ to $6 \mathrm{~cm}$ (mean: 3.4, s.d.: 1.42). Axillary lymph node metastases were present in $55 \%$ of cases, $79 \%$ expressed estrogen receptors (ER), 71\% progestrone receptors (PR) and $22 \%$ overexpressed the Her2/neu receptor (Her2). Details of therapy were not available. Follow-up information was available for up to 19 years: disease-free survival varied between 0 and 12 years (median 5, mean 5.51), while overall survival varied from 0.5 to 19 years (median 7 , mean 6.96).

\section{Expression Analysis}

Expression of the different components of the Akt pathway was studied using immunohistochemistry on consecutive sections of the tissue microarray. Antibodies were selected so as to represent key elements of the different pathway functions (Figure 1). Staining was performed using antibodies to PTEN (PTEN/MMAC1 Ab-2, Neomarkers, Lab Vision Corporation, Fremont, CA, USA; 1:100 dilution), cyclin D1 (Cell Marque Corp., Hot Springs, AR, USA; 1:50 dilution), and phosphorylated (p-)Akt, FKHR, mTOR, and S6 (Cell Signalling Technology, Beverly, MA, USA; 1:50 dilution). FKHR is a member of the Forkhead family of transcription factors and inhibits apoptosis when activated (Figure 1), ${ }^{6}$ while the mammalian target of rapamycin (M-TOR), a serine/threonine kinase, is a central regulator of cell growth and protein synthesis. p70 S6 kinase is a ribosomal protein downstream of mTOR and enhances translation of mRNAs. ${ }^{7}$ Akt is known to activate cyclin D1 by preventing its degradation by activating enzyme kinase glycogen synthase kinase- $3 \beta .^{8}$ Cells from a prostate cancer cell line (PC3) known to contain mutated PTEN and a breast cancer cell line (MCF-7) with wild-type PTEN were formalin fixed and paraffin embedded and used as positive and negative controls. Staining was performed manually in the following manner: deparaffinization; quenching of endogenous peroxidase activity by $3 \%$ solution of hydrogen peroxidase in methanol for $10 \mathrm{~min}$; antigen retrieval for all antibodies except PTEN by boiling slides in $0.01 \mathrm{~mol} / \mathrm{l}$ sodium citrate buffer, $\mathrm{pH} 6.0$, in a vegetable steamer at $100^{\circ} \mathrm{C}$ for $40 \mathrm{~min}$; retrieval for PTEN by vigorously boiling tissue sections for 2 min in citrate buffer, followed by 20 min of slow boiling in a microwave; incubation with the primary antibody for $1 \mathrm{~h}$; secondary antibody for $30 \mathrm{~min}$; signal amplification and chromogen development for $30 \mathrm{~min}$ (wash buffer steps included between each step).

Protein expression was evaluated by two individuals. Discrepancies were resolved by discussion. PTEN staining was scored as 0 when cancer cells showed no staining, while normal cells (glandular epithelium or vascular endothelium) were positive, 1 when staining intensity was less, and 2 when staining intensity was equal to adjacent normal cells (Figure 2). For p-Akt, p-FKHR, p-mTOR, and p-S6 membranous, cytoplasmic, or nuclear staining, and for cyclin D1, only nuclear staining was considered as positive (Figure 3). Staining was graded 0-2 $(0=$ no staining or staining intensity less than normal, $1=$ staining intensity equal to normal, and 
240
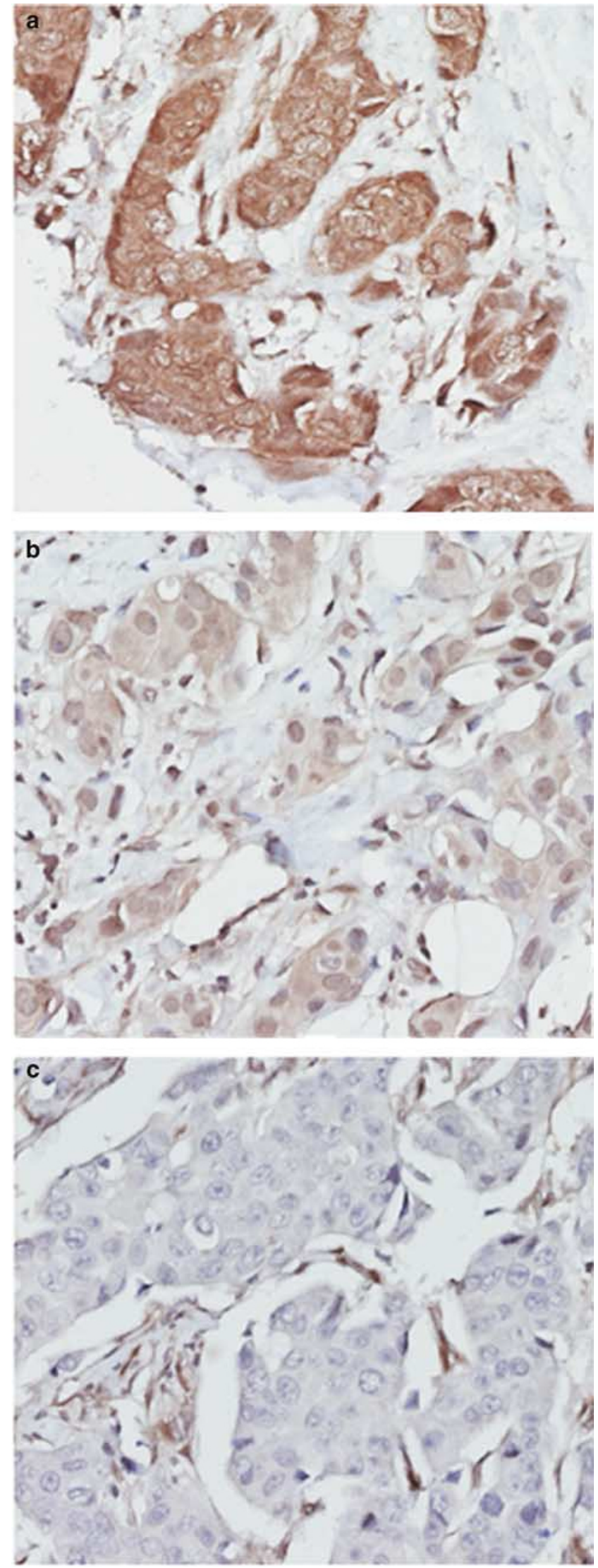

$2=$ strong staining, more than normal). The lowest or highest expression level in any of the cores from a case was recorded as the expression level for that case.

\section{Statistical Analysis}

Within-group correlations of continuous and ordinal variables were assessed using Pearson or Spearman correlation analysis, as appropriate. Comparisons of continuous and ordinal variables between two groups were performed using Student's T-test or the Wilcoxon rank sum test, as appropriate. Twogroup comparisons of dichotomous variables were performed using the Fisher exact test. Multivariable stepwise logistic regression models were used to assess predictors related to dichotomous outcome variables. The Kaplan-Meier method was used to estimate survival and the log-rank and Wilcoxon tests were used to assess survival differences between groups. Multivariable stepwise Cox proportional hazard models were used to assess predictors related to survival. A two-sided $P$-value of 0.05 was considered significant. Analyses were performed using the SAS versions 8.3 and 9.1 (SAS Institute, Cary, NC, USA) statistical software package.

\section{Results}

\section{PTEN Protein Expression}

PTEN protein expression could be evaluated in $81 \%$ $(113 / 140)$ of pure DCIS cases, and in $92 \%(134 / 145)$ of invasive carcinomas. Absence of target lesion in tissue cores and loss of tissue during the sectioning and staining contributed to the reduction in numbers. Complete loss of PTEN expression was observed in $4 \%$ (five cases) of pure DCIS, whereas reduced expression was seen in $7 \%$ (eight cases). Loss of expression correlated significantly with high nuclear grade $(P=0.006)$ and presence of necrosis $(P=0.004)$ (Table 1$)$. Invasive carcinomas showed complete loss of PTEN expression in 15\% (20 cases) and reduced expression in 11\% (15 cases) (Table 2). Loss of expression was associated with high tumor grade (Modified Bloom Richardson composite histologic grade III/III) $(P=0.038)$, greater than 5 mitoses per 10 high-power field $(P=0.037)$, presence of lymphatic invasion $(P=0.062)$, and a high proliferative index $(P=0.04)$. No relationship was noted with ER, PR, or Her 2 (Table 1).

Figure 2 Examples of PTEN protein expression levels observed in invasive breast cancers by immunohistochemistry. (a) Normal expression. Invasive cancer cells with staining intensity equal to normal. (b) Reduced expression. Cancer cells showing staining intensity less than that noted in the adjacent endothelial cells. (c) Loss of expression. No staining is seen in cancer cells while endothelial cells stain normally. 

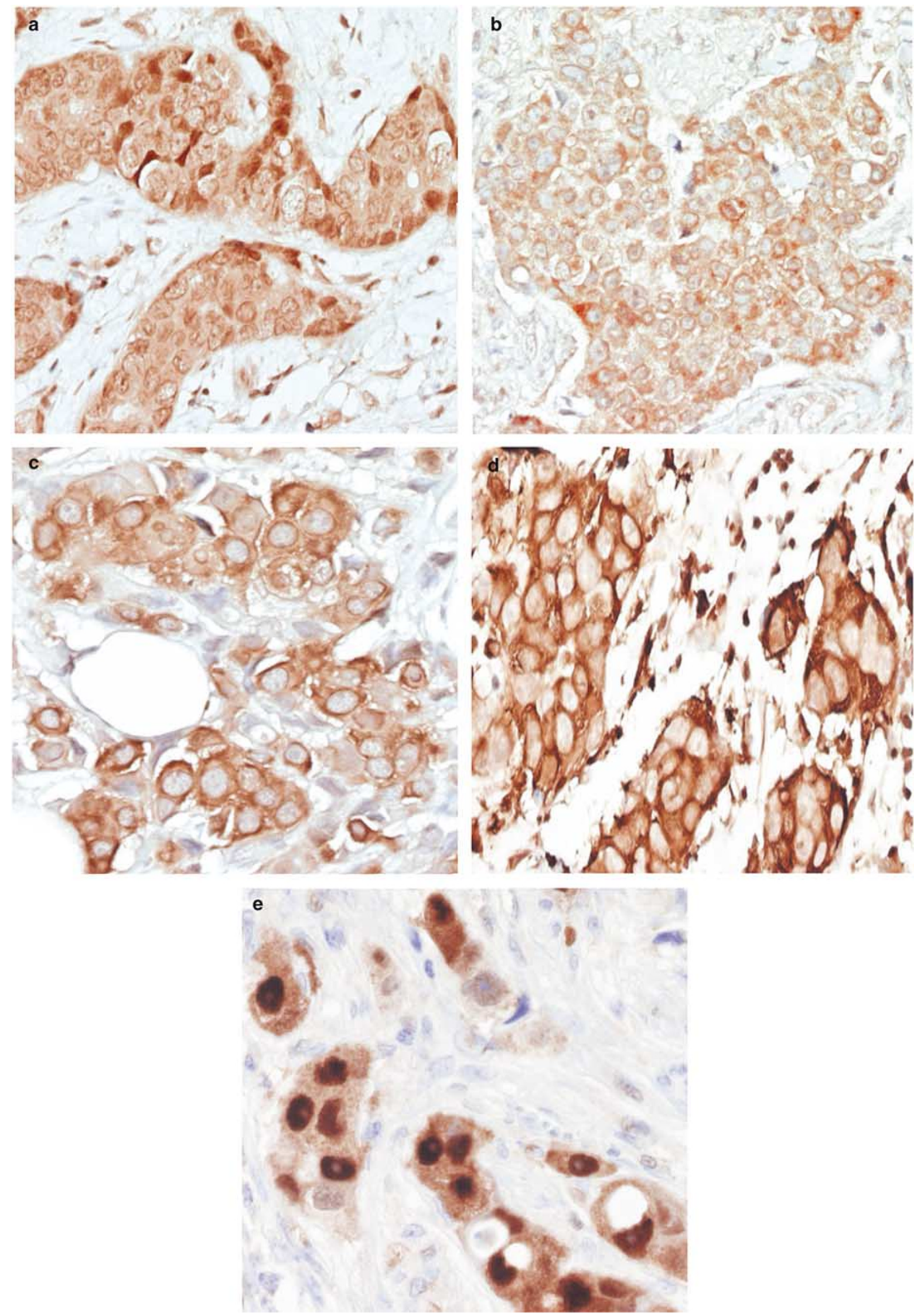

Figure 3 Examples of invasive breast carcinoma showing overexpression of (a) p-AKT, (b) p-FKHR, (c) p-mTOR, (d) p-S6, and (e) cyclin D1. Strong cytoplasmic localization is seen with the phosphorylated antibodies, while cyclin D1 localizes predominantly to the nucleus. 
Table 1 Statistical significance of relationship between tumor characteristics and aberrant expression of the PI3K pathway proteins

\begin{tabular}{|c|c|c|c|c|c|c|c|}
\hline \multirow[t]{2}{*}{ Type of cancer } & \multirow[t]{2}{*}{ Tumor characteristics } & \multicolumn{6}{|c|}{ P-value } \\
\hline & & PTEN & $p-A K T$ & $p-F K H R$ & $p$-mTOR & $p-S 6$ & Bcl1 \\
\hline \multirow[t]{2}{*}{ DCIS } & Nuclear grade & 0.006 & NS & NS & NS & 0.01 & NS \\
\hline & Necrosis & 0.004 & NS & NS & NS & NS & NS \\
\hline \multirow[t]{9}{*}{ Invasive cancer } & Tumor grade & 0.038 & NS & NS & 0.01 & 0.04 & 0.05 \\
\hline & Lymphatic invasion & 0.062 & NS & NS & NS & NS & 0.037 \\
\hline & Axillary metastasis & NS & NS & NS & NS & NS & NS \\
\hline & ER & NS & NS & NS & NS & NS & 0.001 \\
\hline & $\mathrm{PR}$ & NS & NS & NS & NS & NS & 0.04 \\
\hline & Her-2/neu & NS & NS & NS & NS & NS & 0.057 \\
\hline & Ki-67 & 0.04 & NS & NS & NS & NS & NS \\
\hline & Recurrence & 0.085 & NS & NS & 0.028 & NS & 0.002 \\
\hline & Overall survival & NS & NS & NS & NS & NS & NS \\
\hline
\end{tabular}

NS-not significant.

Table 2 Expression of Akt pathway proteins in DCIS and invasive breast cancer

\begin{tabular}{lccc}
\hline Protein & \multicolumn{2}{c}{ Aberrant expression (\% cases) } & \multirow{2}{*}{ Fisher's exact test } \\
\cline { 2 - 3 } & DCIS & Invasive cancer & \\
\hline PTEN & 11 & 26 & $P=0.004$ \\
p-AKT & 33 & 38 & NS \\
p-FKHR & 15 & 20 & NS \\
p-mTOR & 32 & 24 & NS \\
p-S6 & 47 & 72 & NS \\
Cyclin D1 & 60 & 70 & \\
\hline
\end{tabular}

NS-not significant.

Overall, 21\% (8/39) cases with reduced PTEN levels recurred as compared to $14 / 154(9 \%)$ cases without PTEN loss. Although results did not reach statistical significance $(P=0.085)$, a trend of greater recurrence was observed in cases with PTEN protein loss. No statistical difference was noted in survival between women with normal PTEN as compared to those with reduced PTEN.

\section{p-AKT Protein Expression}

Expression of p-AKT was assessed in $81 \%$ (114) of the DCIS cases and $94 \%$ (136) of the invasive cancer cases. Overexpression of p-AKT (more than normal) was observed in 33\% (38/114) of DCIS and in 38\% $(52 / 136)$ of invasive cancers. No significant association was observed with any tumor characteristics, including Her2 and ER expression (Tables 1 and 2).

\section{p-FKHR Protein Expression}

p-FKHR was evaluated in $81 \%$ (114) of the DCIS cases and 94\% (135) of the invasive cancer cases. Overexpression was observed in $15 \%$ (17/114) of DCIS and in $20 \%(27 / 136)$ of invasive carcinomas.
No significant correlation was detected with any tumor characteristics (Tables 1 and 2).

\section{p-mTOR Protein Expression}

p-mTOR expression could be determined in $83 \%$ (116) of DCIS and $82 \%$ (118) of invasive cancer cases. p-mTOR was overexpressed in $32 \%(37 / 116)$ of the DCIS cases and in 24\% (33/138) of the invasive carcinomas. Although no significant relation was observed between p-mTOR and tumor characteristics, invasive carcinomas overexpressing p-mTOR showed a three times greater risk for recurrence $(P=0.03)$ (Tables 1 and 2, Figure 4a).

\section{p-S6 Protein Expression}

p-S6 expression could be evaluated in $83 \%$ (116) of DCIS and 94\% (137) of invasive cancers. A significant difference was noted in the percent of cases overexpressing p-S6 in DCIS cases (47\%; 54/ $116)$ as compared to invasive carcinomas $(72 \%$; 99/137) (Table 2). Additionally, p-S6 overexpression was noted in high-grade DCIS $(P=0.01)$ and poorly differentiated invasive carcinomas $(P=0.04)$ (Table 1).

\section{Cyclin D1 Protein Expression}

Cyclin D1 was evaluated in 74\% (103) of DCIS and $89 \%$ (129) of invasive carcinomas. Overexpression was observed in $60 \%(62 / 103)$ of DCIS and $70 \%$ (90/ 129) of invasive cancers. Overexpression of cyclin D1 was associated with good prognostic features of invasive carcinomas and was more frequently observed in well-differentiated tumors $(P=0.05)$ with low histologic grade $(P=0.008)$, absence of lymphatic invasion $(P=0.037)$, expression of ER $(P=0.001)$, PR $(P=0.04)$, and absence of Her2 overexpression $(P=0.057)$. Disease-free survival 

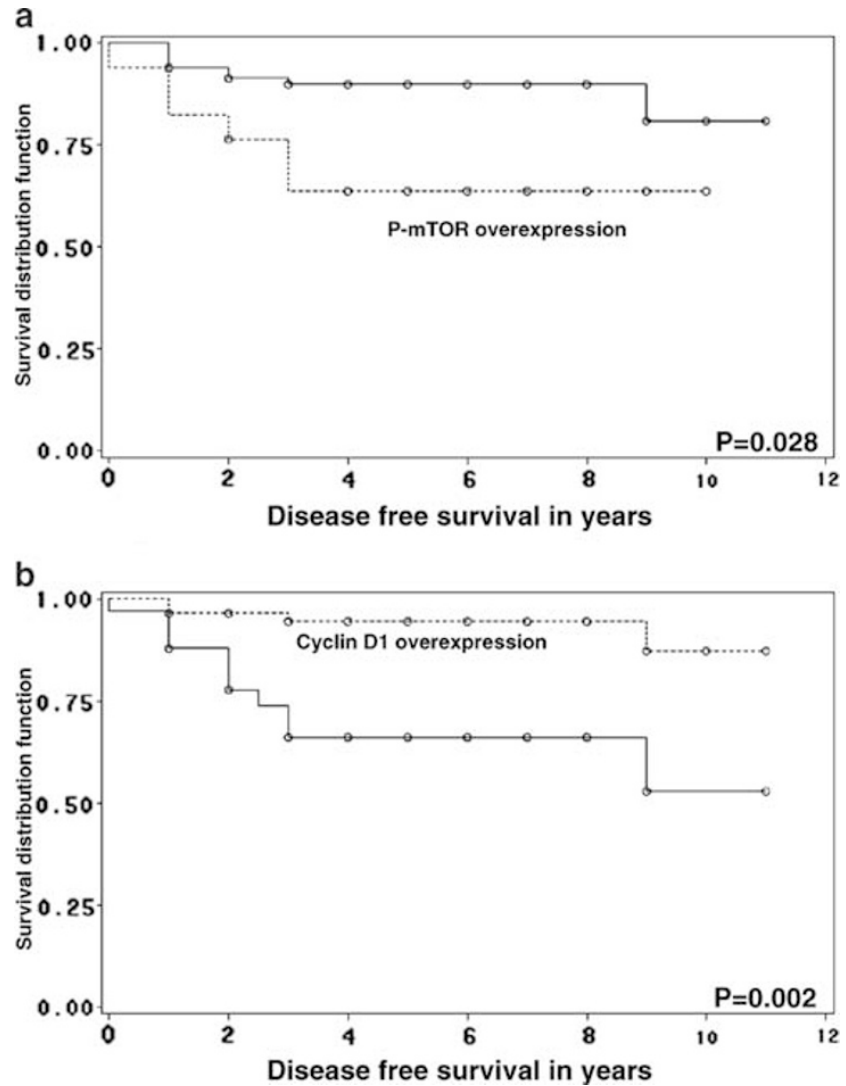

Figure 4 Disease-free survival in invasive cancer cases. Cases stratified by expression levels of (a) p-mTOR and (b) cyclin D1. DFS-disease-free survival in years. Dotted line indicates invasive cancers overexpressing p-mTOR/cyclin D1. Continuous line represents invasive cancers without p-mTOR/cyclin D1 overexpression. Disease-free survival was shorter in cases overexpressing p-mTOR and longer in cases with overexpression of cyclin D1

was longer and patients were less likely to recur (RR $0.17, P=0.002$ ) (Table 1, Figure 4b).

\section{PTEN Loss and pS6 Overexpression Occurs with Progression of DCIS to Invasive Cancer}

Expression of p-AKT, p-FKHR, p-mTOR, and cyclin D1 was similar in pure DCIS and invasive cancer cases (Table 2), indicating that these proteins are overexpressed early in breast cancer and expression levels do not change with disease progression. However, a greater number of invasive cancers showed PTEN loss and p-S6 overexpression than DCIS, thereby suggesting that these proteins may have a role in disease progression from the DCIS to the invasive stage.

\section{Correlations between the Different Proteins}

Protein expression was correlated statistically using the Spearman correlation coefficients (Table 3). PTEN loss correlated with pS6 overexpression only
Table 3 Spearman correlation coefficients

\begin{tabular}{lcrlcr}
\hline & $p$-AKT & $p$-FKHR & $p$-MTOR & $p$-S6 & \multicolumn{1}{c}{$B C l 1$} \\
\hline PTEN & 0.021 & -0.090 & 0.093 & $-0.147^{*}$ & 0.074 \\
p-AKT & & 0.089 & $0.226^{\ddagger}$ & $0.204^{*}$ & 0.003 \\
p-FKHR & & & $0.150^{*}$ & $0.144^{*}$ & $-0.163^{*}$ \\
p-MTOR & & & & 0.013 & -0.037 \\
p-S6 & & & & & -0.035 \\
\hline
\end{tabular}

${ }^{*} P<0.05$

${ }^{\dagger} P<0.01$.

${ }^{\ddagger} P<0.001$.

$(P=0.024)$. Overexpression of $\mathrm{p}-\mathrm{AKT}$ and $\mathrm{p}$-FKHR correlated significantly with overexpression of $\mathrm{p}$ mTOR $(P<0.001,0.019)$ and p-S6 $(P=0.001,0.025)$; however, no correlation was observed between $\mathrm{p}$ AKT and p-FKHR. These findings were confirmed by using the multivariate model.

\section{Discussion}

Using breast cancer tissue microarrays, we have demonstrated that the PI3K pathway can be successfully investigated in vivo with immunohistochemical assays. Our results demonstrate for the first time that the Akt pathway is activated early in breast cancer, in the DCIS stage. Loss of PTEN and pS6 overexpression is associated with disease progression. In contradiction to reported literature, PTEN loss did not correlate with Akt expression in our cases, although other downstream events of the Akt pathway were confirmed with minor differences. mTOR and cyclin D1 were the only proteins whose expression correlated significantly with disease outcome.

We have confirmed our prior observations that PTEN loss is identified in a subset of aggressive breast cancers, and that this loss is associated with disease progression from the in situ to the invasive stage. ${ }^{17,19}$ Experimental data and observations from human cancers have shown that, with the exception of endometrial cancers, the PTEN gene is mutated in advanced and/or metastatic cancers, high-grade gliomas, melanomas, and prostate cancers. ${ }^{20}$ Additionally, introduction of the PTEN gene into cell lines of these cancers results in suppression of metastasis by a yet uncharacterized mechanism. ${ }^{21,22}$ In breast cancer, PTEN loss appears to occur earlier in the disease stage, a situation analogous to that recently reported in testicular cancers, wherein loss of PTEN expression marks the transition from noninvasive intratubular germ cell neoplasias, the presumed precursor lesion of germ cells, to invasive testicular cancers. ${ }^{23}$ The authors studied 60 germ cell tumors of the testis and 22 intratubular germ cell neoplasias for PTEN protein and mRNA expression and found PTEN protein loss in $70 \%$ of testicular tumors, while intratubular germ cell neoplasias contained PTEN protein in all cases. It 
therefore seems reasonable to conclude that, in most human tumors including breast, PTEN plays an important role in cell migration and adhesion. ${ }^{24}$

We did not observe any relation between PTEN loss and Akt overexpression. A similar finding has been observed by Panigrahi et $a l,{ }^{25}$ who, in a study of 670 cases of operable invasive breast cancers, found no correlation between the two. Additionally, it is known that PTEN plays a role in increasing cell motility and spreading by regulating the Rho family GTPases (Rho, Rac1, and Cdc42), which in turn regulate the actin cytoskeleton that is directly responsible for cell motility. It has been shown that cells with targeted deletions of PTEN show increased Cdc42 and Rac1 activity and a resultant increase in motility, thereby providing a link between PTEN loss and tumor invasion. This activity has been shown to occur independent of Akt. $^{26,27}$ Thus, it may be hypothesized that, in breast cancer, PTEN loss activates the Rho family proteins, leading to increased cell migration and invasion through pathways independent of Akt. Further studies are needed for confirmation.

Akt and its downstream proteins, on the other hand, are activated in about $30 \%$ of breast cancers independent of PTEN loss. It is postulated that this may result from activation of other upstream regulators, such as amplification of growth factor receptors. Zhou et $a{ }^{28}$ in a study of 165 invasive breast cancers, noted a positive association between phosphorylated Akt and Her2/neu expression, while Panigrahi et $a l^{25}$ noted a positive significant correlation of p-AKT with ER in their series of cases and therefore suggest that ER, rather than PTEN or Her2, is the upstream regulator. We did not observe either of these correlations.

Our data corroborate the downstream events of Akt activation, which have been described previously. ${ }^{29}$ Akt activation was associated with overexpression of mTOR and S6. Akt is known to be a direct regulator of mTOR. mTOR is emerging as a critical player in growth-factor-controlled translation, and is charged with integrating nutrient and energy homeostasis with mitogenic input. ${ }^{30}$ p-mTOR overexpression was noted in $24 \%$ of our invasive carcinomas and women with these tumors showed greater risk for recurrence and shorter disease-free survival. mTOR inhibitors have been successfully used for treating breast cancers recently. ${ }^{31}$

Experimental models have shown FKHR as downstream of Akt. We did not observe this in our study, although FKHR activation was associated with mTOR and S6 phosphorylation. A similar observation is reported in a tissue-array-based analysis of human prostate cancers. ${ }^{32}$ It is possible that this is a reflection of differences between human tumors and experimental models.

Lastly, our observations with cyclin D1 expression in breast cancer are consistent with that reported in literature and thus further validate the tissue array results. Cyclin D1 is a cell cycle regulator known to play a pivotal role in the development of a subset of human tumors. Overexpression is reported in 30$50 \%$ of primary human breast cancers, is noted early during tumorigenesis, and is associated with a favorable clinical outcome and better response to tamoxifen in ER-alpha-positive tumors. ${ }^{33-35}$ In our series, $60 \%$ of DCIS and $70 \%$ of invasive cancers overexpressed cyclin D1. These cancers expressed good prognostic markers and showed a better clinical outcome. No correlation was observed with PTEN loss or p-AKT overexpression.

Thus, to summarize, pathway analysis using human tumor tissue arrays is a viable alternative for validating experimental data. Our results confirm some of the experimental data, although some significant differences are noted. These may be important in the designing and testing of targeted therapy related clinical trials. Additionally, a significant proportion of breast cancers showed overexpression of cyclin D1 and phosphorylated S6, which raises possibilities for development of these agents as novel targets for chemoprevention.

\section{Acknowledgement}

This study was supported by a research grant (8KB0011) from the California Breast Cancer Research Program.

\section{References}

1 Vivanco I, Sawyers CL. The phosphatidylinositol 3kinase AKT pathway in human cancer. Nat Rev Cancer 2002;2:489-501

2 Luo J, Manning BD, Cantley LC. Targeting the PI3K-Akt pathway in human cancer: rationale and promise. Cancer Cell 2003;4:257-262.

3 Bjornsti MA, Houghton PJ. The TOR pathway: a target for cancer therapy. Nat Rev Cancer 2004;4:335-348.

4 Craven RJ, Lightfoot $H$, Cance WG. A decade of tyrosine kinases: from gene discovery to therapeutics. Surg Oncol 2003;12:39-49.

5 White MF. The IRS-signalling system: a network of docking proteins that mediate insulin action. Mol Cell Biochem 1998;182:3-11.

6 Dijkers PF, Medema RH, Lammers JW, et al. Expression of the pro-apoptotic Bcl-2 family member Bim is regulated by the forkhead transcription factor FKHRL1. Curr Biol 2000;10:1201-1204.

7 Nave BT, Ouwens M, Withers DJ, et al. Mammalian target of rapamycin is a direct target for protein kinase B: identification of a convergence point for opposing effects of insulin and amino-acid deficiency on protein translation. Biochem J 1999;344(Part 2):427-431.

8 Diehl JA, Cheng M, Roussel MF, et al. Glycogen synthase kinase-3beta regulates cyclin D1 proteolysis and subcellular localization. Genes Dev 1998;12: 3499-3511.

$9 \mathrm{Li}$ J, Yen C, Liaw D, et al. PTEN, a putative protein tyrosine phosphatase gene mutated in human brain, 
breast, and prostate cancer. Science 1997;275: 1943-1947.

10 Stambolic V, Suzuki A, de la Pompa JL, et al. Negative regulation of $\mathrm{PKB} / \mathrm{Akt}$-dependent cell survival by the tumor suppressor PTEN. Cell 1998;95:29-39.

11 Staal SP. Molecular cloning of the akt oncogene and its human homologues AKT1 and AKT2: amplification of AKT1 in a primary human gastric adenocarcinoma. Proc Natl Acad Sci USA 1987;84:5034-5037.

12 Cheng JQ, Ruggeri B, Klein WM, et al. Amplification of AKT2 in human pancreatic cells and inhibition of AKT2 expression and tumorigenicity by antisense RNA. Proc Natl Acad Sci USA 1996;93:3636-3641.

13 Ma YY, Wei SJ, Lin YC, et al. PIK3CA as an oncogene in cervical cancer. Oncogene 2000;19:2739-2744.

14 Philp AJ, Campbell IG, Leet C, et al. The phosphatidylinositol $3^{\prime}$-kinase p85alpha gene is an oncogene in human ovarian and colon tumors. Cancer Res 2001;61:7426-7429.

15 Bachman KE, Argani P, Samuels Y, et al. The PIK3CA gene is mutated with high frequency in human breast cancers. Cancer Biol Ther 2004;3:772-775.

16 Mutter GL, Lin MC, Fitzgerald JT, et al. Altered PTEN expression as a diagnostic marker for the earliest endometrial precancers. J Natl Cancer Inst 2000;92: 924-930.

17 Bose S, Crane A, Hibshoosh $\mathrm{H}$, et al. Reduced expression of PTEN correlates with breast cancer progression. Hum Pathol 2002;33:405-409.

18 Nagata Y, Lan KH, Zhou X, et al. PTEN activation contributes to tumor inhibition by trastuzumab, and loss of PTEN predicts trastuzumab resistance in patients. Cancer Cell 2004;6:117-127.

19 Bose S, Wang SI, Terry MB, et al. Allelic loss of chromosome 10q23 is associated with invasion in breast carcinomas. Oncogene 1998;17:123-127.

20 Ali IU, Schriml LM, Dean M. Mutational spectra of PTEN/MMAC1 gene: a tumor suppressor with lipid phosphatase activity. J Natl Cancer Inst 1999;91: 1922-1932.

21 Davies MA, Kim SJ, Parikh NU, et al. Adenoviralmediated expression of MMAC/PTEN inhibits proliferation and metastasis of human prostate cancer cells. Clin Cancer Res 2002;8:1904-1914.

22 Hwang PH, Yi HK, Kim DS, et al. Suppression of tumorigenicity and metastasis in B16F10 cells by PTEN/MMAC1/TEP1 gene. Cancer Lett 2001;172: 83-91.

23 Di Vizio D, Cito L, Boccia A, et al. Loss of the tumor suppressor gene PTEN marks the transition from intratubular germ cell neoplasias (ITGCN) to invasive germ cell tumors. Oncogene 2005;24:1882-1894.

24 Tamura M, Gu J, Matsumoto $\mathrm{K}$, et al. Inhibition of cell migration, spreading, and focal adhesions by tumor suppressor PTEN. Science 1998;280:16141617.

25 Panigrahi AR, Pinder SE, Chan SY, et al. The role of PTEN and its signalling pathways, including AKT, in breast cancer; an assessment of relationships with other prognostic factors and with outcome. J Pathol 2004;204:93-100.

26 Liliental J, Moon SY, Lesche R, et al. Genetic deletion of the Pten tumor suppressor gene promotes cell motility by activation of Rac1 and Cdc42 GTPases. Curr Biol 2000;10:401-404.

27 Welch HC, Coadwell WJ, Ellson CD, et al. P-Rex1, a PtdIns(3,4,5)P3- and Gbetagamma-regulated guaninenucleotide exchange factor for Rac. Cell 2002;108: 809-821.

28 Zhou X, Tan M, Stone Hawthorne V, et al. Activation of the $\mathrm{Akt} / \mathrm{mammalian}$ target of rapamycin/4E-BP1 pathway by ErbB2 overexpression predicts tumor progression in breast cancers. Clin Cancer Res 2004;10 $6779-6788$.

29 Aoki M, Blazek E, Vogt PK. A role of the kinase mTOR in cellular transformation induced by the oncoproteins P3k and Akt. Proc Natl Acad Sci USA 2001;98: 136-141.

30 Bader AG, Vogt PK. An essential role for protein synthesis in oncogenic cellular transformation. Oncogene 2004;23:3145-3150.

31 deGraffenried LA, Friedrichs WE, Russell DH, et al. Inhibition of mTOR activity restores tamoxifen response in breast cancer cells with aberrant Akt Activity. Clin Cancer Res 2004;10:8059-8067.

32 Thomas GV, Horvath S, Smith BL, et al. Antibodybased profiling of the phosphoinositide 3-kinase pathway in clinical prostate cancer. Clin Cancer Res 2004;10:8351-8356.

33 Hosokawa Y, Arnold A. Mechanism of cyclin D1 (CCND1, PRAD1) overexpression in human cancer cells: analysis of allele-specific expression. Genes Chromosomes Cancer 1998;22:66-71.

34 Weinstein IB. Relevance of cyclin D1 and other molecular markers to cancer chemoprevention. J Cell Biochem Suppl 1996;25:23-28.

35 Han S, Park K, Bae BN, et al. Cyclin D1 expression and patient outcome after tamoxifen therapy in estrogen receptor positive metastatic breast cancer. Oncol Rep 2003;10:141-144. 